\title{
Reseña de: Remedios Álvarez, María José Clavo, Olaya Fernández y María Ángeles Goicoechea. Maternidad lesbiana: Del deseo a la realidad. Barcelona-Madrid: Editorial Egales, S.L., 2018.
}

\author{
Carlos ÁlVAREZ FERNÁNDEZ \\ Universidad del País Vasco
}

El interés por los estudios acerca de la maternidad lesbiana ha sido fruto de la confluencia de dos factores fundamentales. Por un lado, la legislación a favor de los derechos homosexuales y de las mujeres, teniendo como hito la ley de 2005 de matrimonio homosexual y la ley de 2006 sobre técnicas de reproducción asistida. Por otro lado, un cambio de mentalidad positiva en torno a la homosexualidad en general y a la constitución de nuevos tipos de familias, entre ellas la lesboparental, ha ido ocupando un lugar cada vez más importante. De esta forma hemos asistido a la proliferación creciente de investigaciones en torno a la idoneidad o no de los matrimonios igualitarios, así como sobre su impacto en la educación de los hijos e hijas de estas nuevas familias.

En este panorama, la investigación planteada por las cuatro autoras -pertenecientes al Grupo de Investigación Igualdad y Género, de la Universidad de La Rioja- sobre la maternidad lesbiana resulta una contribución esencial. No sólo en tanto que viene a llenar el vacío existente de estudios sobre el colectivo de mujeres lesbianas, sino también por aportar una visión más compleja sobre la maternidad en general. Los distintos perfiles académicos de las coautoras permiten realizar una aproximación multidisciplinar, en la que se combinan planteamientos procedentes de la historia contemporánea, la filosofía, la sociología o la educación.

Los contenidos de la revista se publican bajo una licencia de Creative Commons Reconocimiento 4.0 Internacional (CC BY 4.0)

Feminismo/s 33, junio 2019, pp. 351-354 
Reseña de: Remedios Álvarez, María José Clavo, Olaya Fernández y María Ángeles Goicoechea. Maternidad lesbiana: Del deseo a la realidad. Barcelona-Madrid: Editorial Egales, S.L., 2018.

El trabajo ahonda en terrenos poco explorados como las representaciones y estereotipos que se construyen en España en torno a las familias homoparentales. Para los estudios en perspectiva de género, esta obra presenta una demostración práctica de las potencialidades de las herramientas de la teoría feminista a la hora de abordar la relación entre la feminidad y la maternidad. El aparato teórico metodológico se construye a partir de referencias múltiples, entre las que ocupa un lugar central la teorización del patriarcado que realiza Pierre Bourdieu en su obra La dominación masculina, así como las aportaciones de distintas autoras feministas, como Luisa Muraro, Adrienne Rich o Simone de Beauvoir, entre otras.

Esta obra, además, supone una recopilación de una bibliografía extensa, siendo así una de las principales contribuciones a la hora de situar al lector en la materia. A nivel de metodología, se combina el estudio documental con la realización de entrevistas orales, tanto a mujeres lesbianas como a especialistas sanitarios. De esta forma se ofrece una visión compleja, combinando las voces de investigadores/as con las voces de las propias mujeres y del personal médico. El enfoque cualitativo y la importancia del lenguaje como medio de acceder a la experiencia es central aquí, puesto que es el que vuelve inteligibles y coherentes los recuerdos.

El libro presenta tres partes principales: la primera de ellas se centra en una reflexión sobre los conceptos de familia, matrimonio y maternidad, que ocupan los dos primeros capítulos. Para ello se parte de una visión construccionista, que analiza estas estructuras desde un punto de vista social, legal e histórico, huyendo de visiones naturalizadoras. De esta forma, las autoras advierten la existencia de múltiples modelos de familias - entre ellas la familia homoparental- que han sido legalmente reconocidas en los últimos años, desplazando a la «familia tradicional» como única forma de articular las relaciones de parentesco en la sociedad. Por otro lado, se plantea la importancia del matrimonio como vía de reconocimiento de derechos para las madres lesbianas. Y se concluye este apartado preguntándose los límites de la elección de las mujeres por la maternidad partiendo de la base teórica de la existencia de la «dominación masculina» y la constitución de regímenes de poder heterosexistas, que históricamente han restado agencia a las mujeres a la hora de decidir sobre su propio cuerpo. En cualquier caso, no existe un consenso dentro del feminismo a la hora de determinar la relación entre

Feminismo/s 33, junio 2019, pp. 351-354 
Reseña de: Remedios Álvarez, María José Clavo, Olaya Fernández y María Ángeles Goicoechea. Maternidad lesbiana: Del deseo a la realidad. Barcelona-Madrid: Editorial Egales, S.L., 2018.

feminidad y maternidad, habiendo actitudes enfrentadas a este respecto por no dar cuenta de la heterogeneidad de la propia feminidad.

La segunda parte del libro se desarrolla en los capítulos tres y cuatro. Las autoras se centran en el trabajo de campo realizado con las madres lesbianas, así como con los profesionales sanitarios de la ginecología y obstetricia. En líneas generales, las madres lesbianas han tenido que afrontar una serie de obstáculos. En primer lugar, los prejuicios existentes en parte de la sociedad, que colocan muchas veces a estas mujeres en la tesitura de elegir entre su deseo sexual y el maternal. Por otro lado, se encuentran con las dificultades materiales por lo costoso de las gestiones para convertirse en madres. Otro de los problemas viene derivado del propio proceso de gestación, ya que se trata de una decisión a la que las parejas heterosexuales no tienen que hacer frente. La maternidad lesbiana presenta rupturas y continuidades con respecto al modelo de maternidad tradicional. Las rupturas vienen dadas por la constitución de familias encabezadas por personas del mismo sexo y por la ausencia de un progenitor masculino, aunque en ciertos casos sea una figura secundaria. Las continuidades se observan en la reproducción de la estructura del matrimonio nuclear y la preferencia por los lazos consanguíneos con los hijos e hijas. En relación al personal sanitario, las autoras han podido observar, a través de este estudio, un proceso creciente de normalización y aceptación de la diversidad afectivo-sexual y hacia los nuevos tipos de parejas y de familias. Un cambio de mentalidad relacionado con la irrupción de una legislación favorable hacia el colectivo homosexual.

La tercera parte se subdivide en varios capítulos. En el capítulo cinco, se analiza la evolución de las opiniones de la sociedad española hacia la homosexualidad en general y hacia las madres lesbianas en particular. Uno de los principales defectos que se detecta en el análisis de las encuestas es el uso del masculino universalizador, que invisibiliza a las mujeres lesbianas. Las estadísticas muestran la coexistencia del machismo y la homofobia, sufriendo las mujeres en muchas ocasiones una doble discriminación. Con todo, los últimos años reflejan un descenso en la valoración negativa de las madres lesbianas. El sexto capítulo se centra en la importancia de la socialización y la educación como herramientas para destruir los estereotipos negativos sobre la maternidad lesbiana. Con todo, la lucha por la visibilidad se tropieza muchas veces con obstáculos que a nivel micro reflejan la existencia de la

Feminismo/s 33, junio 2019, pp. 351-354 
norma heterosexista. Por ejemplo, señalan la existencia del Día del Padre y de la Madre y no de la Familia, un término más inclusivo. En el capítulo siete, las autoras parten de una reflexión acerca de si se ha conseguido de forma efectiva la igualdad. A este respecto señalan cómo los formularios de la administración pública siguen sin ofrecer, en muchas ocasiones, modelos de parentalidad alternativos al tradicional. Además de la propia inestabilidad de la reproducción asistida que en sus escasos años de existencia ha conocido avances y retrocesos.

En definitiva, nos encontramos ante una obra de referencia para la comprensión de la maternidad lesbiana en todo el territorio español. Se trata de un manual de gran utilidad social, que puede servir al colectivo LGTBIQ como una herramienta en el camino hacia la igualdad real. Su redacción facilita que su público potencial se amplíe más allá del ámbito universitario y sanitario, siendo fácilmente accesible a todo tipo de personas de la sociedad en general. 